\title{
Projectively Flat Fourth Root Finsler Metrics
}

\author{
Benling Li*and Zhongmin Shen ${ }^{\dagger}$
}

\section{Introduction}

One of important problems in Finsler geometry is to study the geometric properties of locally projectively flat Finsler manifolds. Locally projectively flat metrics are of scalar flag curvature, namely, the flag curvature is a scalar function of tangent vectors, independent of the tangent planes containing the tangent vector.

A Finsler metric on an open domain in $R^{n}$ is said to be projectively flat if its geodesics are straight lines. Hilbert's Fourth Problem in the regular case is to study and characterize projectively flat metrics on a convex open domain in $R^{n}$. Projectively flat Riemannian metrics are those of constant sectional curvature $\mathbf{K}=\mu$, which can be expressed in the following form on an appropriate ball in $R^{n}$ :

$$
F=\frac{\sqrt{|y|^{2}+\mu\left(|x|^{2}|y|^{2}-\langle x, y\rangle^{2}\right)}}{1+\mu|x|^{2}} .
$$

There are many non-Riemannian projectively flat Finsler metrics. For example, the well-known Funk metric and Hilbert metric on a strongly convex domain are projectively flat. These metrics even have constant flag curvature. See [1] for more information on projectively flat Finsler metrics of constant flag curvature. In [2], we study and characterize projectively flat $(\alpha, \beta)$-metrics which are defined by a Riemannian metric $\alpha=\sqrt{a_{i j}(x) y^{i} y^{j}}$ and a 1 -form $\beta=$ $b_{i}(x) y^{i}$. Two special examples are given as follows:

$$
\begin{aligned}
& F=\frac{\sqrt{|y|^{2}-\left(|x|^{2}|y|^{2}-\langle x, y\rangle^{2}\right)}}{1-|x|^{2}}+\frac{\langle x, y\rangle}{1-|x|^{2}} \\
& F=\frac{\left(\sqrt{|y|^{2}-\left(|x|^{2}|y|^{2}-\langle x, y\rangle^{2}\right)}+\langle x, y\rangle\right)^{2}}{\left(1-|x|^{2}\right)^{2} \sqrt{|y|^{2}-\left(|x|^{2}|y|^{2}-\langle x, y\rangle^{2}\right)}} .
\end{aligned}
$$

The metric in (2) is the Funk metric in a unit ball $B^{n}(1) \subset R^{n}$ and the metric in (3) is constructed by L. Berwald [3].

\footnotetext{
*supported in part by NNSFC (10801080), Municipal Natural Science Foundation of Ningbo (2008A610014) and sponsored by K.C.Wong Magna Fund in Ningbo University

†Supported in part by NNSF of China(10671214) and NSF (DMS-0810159)
} 
There are two important classes of reversible Finsler metrics.

$$
\begin{aligned}
& F=A^{1 / 4} \\
& F=\left(A^{1 / 2}+B\right)^{1 / 2}
\end{aligned}
$$

where $A=a_{i j k l}(x) y^{i} y^{j} y^{k} y^{l}$ and $B=b_{i j}(x) y^{i} y^{j}$. A Finsler metric in the form (4) is called a fourth root metric and a Finsler metric in the form (5) is called a generalized fourth root metric. When $A$ is a perfect square of a quadratic form, $F=A^{1 / 4}$ is just a Riemannian metric. Thus (generalized) fourth root metrics include Riemannian. Similarly, one can consider $m$-th root metrics and their generalizations. In four-dimension, the special fourth root metric in the form $F=\sqrt[4]{y^{1} y^{2} y^{3} y^{4}}$ is called the Berwald-Moore metric. This metric is singular in $y$ and not positive definite. Recently, physicists are interested in fourth-root of metrics [6]. Thus it is important to study the geometric properties of fourth root metrics.

A Finsler metric $F=F(x, y)$ is said to be locally Minkowskian if at every point, there is a local coordinate domain in which the metric $F=F(y)$ is independent of its position $x$. In this case, all geodesics are linear lines $x^{i}(t)=$ $t a^{i}+b^{i}$. A Finsler metric $F=F(x, y)$ is said to be locally projectively flat if at every point, there is a local coordinate domain in which the geodesics are straight lines, namely, $x^{i}(t)=f(t) a^{i}+b^{i}$. The main purpose of this paper is to study locally projectively flat fourth root metrics and its generalized metrics. We prove the following

Theorem 1.1 Let $F=A^{1 / 4}$ be a fourth root metric on a manifold of dimension $n \geq 3$. Assume that $A$ is irreducible. If $F$ is locally projectively flat, then it is locally Minkowskian.

If $A=\left(a_{i j}(x) y^{i} y^{j}\right)^{2}$ is the square of a Riemannian metric of constant sectional curvature $\mathbf{K}=\mu$, then $F=A^{1 / 4}=\sqrt{a_{i j}(x) y^{i} y^{j}}$ is locally projectively flat. But it is not locally Minkowskian when $\mu \neq 0$. Thus the condition on the irreducibility condition of $A$ can not be removed although it might be slightly weakenned.

Theorem 1.2 Let $F=\left(A^{1 / 2}+B\right)^{1 / 2}$ be a generalized fourth root metric on a manifold of dimension $n \geq 3$. Assume that $A$ and $A-B^{2}$ are both irreducible and $B \neq 0$. If $F$ is locally projectively flat, then it is locally Minkowskian.

The following example shows that the irreducibility condition on $A-B^{2}$ can not be dropped although it might be slightly weakended.

Example 1.1 Let $F=\left(A^{1 / 2}+B\right)^{1 / 2}$ be a fourth root metric on $B^{n} \subset R^{n}$ defined by

$$
\begin{aligned}
A: & =\frac{|y|^{4}+\left(|x|^{2}|y|^{2}-\langle x, y\rangle^{2}\right)^{2}}{4\left(1+|x|^{4}\right)^{2}} \\
B: & =\frac{\left(1+|x|^{4}\right)|x|^{2}|y|^{2}+\left(1-|x|^{4}\right)\langle x, y\rangle^{2}}{2\left(1+|x|^{4}\right)^{2}}
\end{aligned}
$$


Note that

$$
A-B^{2}=\left[\frac{-2|x|^{2}\langle x, y\rangle^{2}+\left(1+|x|^{4}\right)|y|^{2}}{2\left(1+|x|^{4}\right)^{2}}\right]^{2} .
$$

Thus $A-B^{2}$ is reducible. $F$ is projectively flat, but not locally Minkowskian.

The classification of projectively flat generalized fourth root metrics without assumption on the irreducibility has not been done yet. Certainly, this is an interesting problem which needs attention.

\section{Preliminaries}

Let $F$ be a Finsler metric on a manifold $M$. We always assume that $F$ is positive definite (or strongly convex), namely, the matrix $g_{i j}=g_{i j}(x, y)$ is positive definite, where

$$
g_{i j}(x, y):=\frac{1}{2}\left[F^{2}\right]_{y^{i} y^{j}}(x, y), \quad(y \neq 0) .
$$

The geodesics of $F$ are characterized by a system of equations:

$$
\frac{d^{2} x^{i}}{d t^{2}}+2 G^{i}\left(x, \frac{d x}{d t}\right)=0
$$

where

$$
G^{i}=\frac{1}{4} g^{i l}\left\{\left[F^{2}\right]_{x^{m} y^{l}} y^{m}-\left[F^{2}\right]_{x^{l}}\right\} .
$$

Clearly, if $F$ is Riemannian, then $G^{i}=G^{i}(x, y)$ are quadratic in $y$. $F$ is called a Berwald metric if $G^{i}=G^{i}(x, y)$ are quadratic in $y$. It is called a Landsberg metric if

$$
F_{y^{i}}\left[G^{i}\right]_{y^{j} y^{k} y^{l}}=0 .
$$

Thus every Riemannian metric is a Berwald metric and every Berwald metric is a Landsberg metric.

For a Finsler metric, the Riemann curvature $\mathbf{R}_{y}: T_{x} M \rightarrow T_{x} M$ is defined by $\mathbf{R}_{y}(u)=R_{k}^{i}(x, y) u^{k} \frac{\partial}{\partial x^{i}} x, u=\left.u^{k} \frac{\partial}{\partial x^{k}}\right|_{x}$, where

$$
R_{k}^{i}(x, y):=2 \frac{\partial G^{i}}{\partial x^{k}}-y^{j} \frac{\partial^{2} G^{i}}{\partial x^{j} \partial y^{k}}+2 G^{j} \frac{\partial^{2} G^{i}}{\partial y^{j} \partial y^{k}}-\frac{\partial G^{i}}{\partial y^{j}} \frac{\partial G^{j}}{\partial y^{k}} .
$$

For each tangent plane $\Pi \subset T_{x} M$ and $y \in P$, the flag curvature of ( $\left.\Pi, y\right)$ is defined by

$$
\mathbf{K}(\Pi, y):=\frac{g_{i m}(x, y) R_{k}^{i}(x, y) u^{k} u^{m}}{F)\left(x, y^{2} g_{i j}(x, y) u^{i} u^{j}-\left[g_{i j}(x, y) y^{i} u^{j}\right]^{2}\right.},
$$

where $u \in \Pi$ such that $\Pi=\operatorname{span}\{y, u\}$. There is a large class of Finsler metrics whose flag curvature $\mathbf{K}(\Pi, y)=K(x, y)$ is independent of tangent planes $\Pi$ containing $y \in T_{x} M$. Such metrics are said to be of scalar flag curvature. When the metric is Riemannian, the flag curvature $\mathbf{K}(\Pi, y)=K(\Pi)$ is independent of 
$y \in T_{x} M$. Thus it is of scalar flag curvature $\mathbf{K}=K(x, y)$ if and only if is of isotropic sectional curvature $\mathbf{K}=K(x)$ (constant if dimension $\geq 3$ ). We have the following important theorem.

Theorem 2.1 (Numata [5]) Let $F$ be a Landsberg metric of scalar flag curvature on a manifold of dimension $n \geq 3$. If the flag curvature $\mathbf{K} \neq 0$, then it is Riemannian.

On the other hand, we have the following well-known theorem.

Theorem 2.2 Every Berwald metric with $\mathbf{K}=0$ is locally Minkowskian.

A simple fact is that a Finsler metric $F=F(x, y)$ on an open subset $\mathcal{U} \subset R^{n}$ is projectively flat if and only if the spray coefficients are in the form $G^{i}=P y^{i}$. It is equivalent to the following Hamel equation

$$
F_{x^{m} y^{k}} y^{m}=F_{x^{k}} .
$$

In this case, $P=F_{x^{m}} y^{m} /(2 F)$ and the metric is of scalar flag curvature given by

$$
\mathbf{K}=\frac{P^{2}-P_{x^{m}} y^{m}}{F^{2}}
$$

Thus, locally projectively flat Finsler metrics are of scalar flag curvature.

Let us consider the special case when $G^{i}=P y^{i}$ where $P=P_{i}(x) y^{i}$ is a local 1 -form. Assume that the dimension $n \geq 3$. Let

$$
U:=\left\{x \in M \mid \mathbf{K}(x, y) \neq 0 \text { for some } y \in T_{x} M\right\} .
$$

Assume that $U \neq \emptyset$. By Theorem 2.1, $F$ is Riemannian on $U$ with $\mathbf{K}=$ constant $\neq 0$. By continuity, one can easily conclude that $U=M$, namely, $F$ is Riemannian on the whole manifold. Assume that $U=\emptyset$, i.e., $\mathbf{K}=0$ on $M$. Since $F$ is a Berwald metric, it must be locally Minkowskian.

The two-dimensional case is solved by L. Berwald.

Theorem 2.3 Berwald ([4]) Let $F$ be a locally projectively flat Landsberg metric on a surface $M$. Then it is either Riemannian with non-zero constant Gauss curvature or locally Minkowskian.

If one allows singular metrics in Theorem 2.3, then besides the above two cases, the metric might be a Berwald metric which can be locally expressed in the following form

$$
F=\left(y^{1}+f\left(x^{1}, x^{2}\right) y^{2}\right)^{4} /\left(y^{2}\right)^{2},
$$

where $f=f\left(x^{1}, x^{2}\right)$ is a function satusfies $x^{1}+x^{2} f=\psi(f)$ for some function $\psi$ with $\psi^{\prime \prime} \neq 0$. 
We now consider fourth root metrics. Let $A(x, y):=a_{i j k l}(x) y^{i} y^{j} y^{k} y^{l}$ be a homogeneous polynomial of degree four on tangent spaces. Assume that $A=$ $A(x, y)>0$ for any $y \neq 0$. Then the Hessian $g_{i j}:=\frac{1}{2}\left[F^{2}\right]_{y^{i} y^{j}}$ is given by

$$
g_{i j}=\frac{1}{8} A^{-3 / 2}\left\{2 A A_{i j}-A_{i} A_{j}\right\},
$$

where

$$
A_{i}:=\frac{\partial A}{\partial y^{i}}, \quad A_{i j}:=\frac{\partial^{2} A}{\partial y^{i} \partial y^{j}} .
$$

Thus if $2 A A_{i j}-A_{i} A_{j}$ is positive definite, then $F$ is a positive definite Finsler metric.

Example 2.1 Let

$$
A:=\sum_{i=1}^{n}\left(y^{i}\right)^{4} .
$$

For some directions, say, $y=(1,0, \cdots, 0)$, $\operatorname{det}\left(g_{i j}\right)=0$. Thus $F=A^{1 / 4}$ is not positive definite.

Example 2.2 Let $A$ be a two-dimensional homogenous polynomial of degree four in the following form:

$$
A=\left(y^{1}\right)^{4}+2 c\left(y^{1}\right)^{2}\left(y^{2}\right)^{2}+\left(y^{2}\right)^{4} .
$$

It is easy to show that $F=A^{1 / 4}$ is positive definite if and only if $1<c<3$.

Example 2.3 Let $\alpha:=\sqrt{a_{i j} y^{i} y^{j}}$ be an Euclidean norm and $\beta:=b_{i} y^{i}$ be a 1 -form on $R^{n}$ with $b:=\sqrt{a^{i j} b_{i} b_{j}}<b_{o}$. Then

$$
A:=\alpha^{4}+\varepsilon \alpha^{2} \beta^{2}+\beta^{4}
$$

is strongly convex if

$$
\begin{gathered}
1+\varepsilon s^{2}+s^{4}>0, \quad\left(|s|<b_{o}\right) \\
4+2 \varepsilon \rho^{2}+\left(4 \varepsilon+12 \rho^{2}-\rho^{2} \varepsilon^{2}\right) s^{2}+\left(2 \rho^{2} \varepsilon-8+3 \varepsilon^{2}\right) s^{4}>0, \quad\left(|s| \leq \rho<b_{o}\right) .
\end{gathered}
$$

\section{$3 \quad$ Projectively flat fourth root metrics}

In this section, we are going to discuss projectively flat fourth root metrics $F=A^{1 / 4}$ on an open subset $\mathcal{U} \subset R^{n}$. For simplicity, we let

$$
A_{0}:=A_{x^{m}} y^{m}, \quad A_{00}:=A_{x^{p} x^{q}} y^{p} y^{q} .
$$

First we have the following 
Lemma 3.1 Let $F=A^{1 / 4}$ be a fourth root metric on an open subset $\mathcal{U} \subset R^{n}$. It is projectively flat if and only if

$$
4 A\left(A_{x^{m} y^{k}} y^{m}-A_{x^{k}}\right)=3 A_{0} A_{y^{k}} .
$$

Proof: (8) follows from (6) immediately.

Q.E.D.

Proof of Theorem 1.1: Assume that $F$ is projectively flat. Since $A$ is irreducible and $\operatorname{deg}\left(A_{y^{k}}\right)=3$, by (8), one can conclude that $A_{0}$ is divisible by $A$, that is, there is a 1 -form $\eta$ such that

$$
A_{0}=8 \eta A \text {. }
$$

Thus the spray coefficients $G^{i}=P y^{i}$ are given by

$$
P=\frac{A_{0}}{8 A}=\eta \text {. }
$$

We see that $G^{i}=\eta y^{i}$ are quadratic in $y$. Therefore $F$ is a Berwald metric.

Assume that $n \geq 3$. By Theorem 2.1, if $\mathbf{K} \neq 0$, then $F$ is Riemannian. Thus $A$ is a perfect square of a Riemannian metric. This contradicts our assumption. Thus $\mathbf{K}=0$. That is, $F$ is a Berwald metric with $\mathbf{K}=0$. Therefore, $F$ is locally Minkowskian.

Q.E.D.

\section{Generalized Fourth Root Metrics}

In this section, we shall consider the generalized fourth root metrics

$$
F=\left(A^{1 / 2}+B\right)^{1 / 2},
$$

where $A=a_{i j k l}(x) y^{i} y^{j} y^{k} y^{l}$ and $B=b_{i j}(x) y^{i} y^{j}$. We denote $A_{0}$ and $A_{00}$ as above and let $B_{0}:=B_{x^{m}} y^{m}$ and $B_{00}=B_{x^{p} x^{q}} y^{p} y^{q}$.

Let

$$
X_{k}:=\frac{B A_{y^{k}}-2 A B_{y^{k}}}{2\left(B^{2}-A\right)}, \quad Y_{k}:=\frac{2 B B_{y^{k}}-A_{y^{k}}}{4\left(B^{2}-A\right)} .
$$

Lemma 4.1 Let $F=\left(A^{1 / 2}+B\right)^{1 / 2}$ be a generalized fourth root metric on a domain in $R^{n}$. Assume that $A=a_{i j k l}(x) y^{i} y^{j} y^{k} y^{l}$ is irreducible. A generalized fourth root metric $F=\left(A^{1 / 2}+B\right)^{1 / 2}$ is projectively flat on an open domain in $R^{n}$ if and only if

$$
\begin{aligned}
& A_{x^{m} y^{k}} y^{m}-A_{x^{k}}=\frac{A_{y^{k}}}{2 A} A_{0}+Y_{k} A_{0}+X_{k} B_{0} \\
& B_{x^{m} y^{k}} y^{m}-B_{x^{k}}=\frac{1}{4 A} X_{k} A_{0}+Y_{k} B_{0} .
\end{aligned}
$$


Proof: By a direct computation, we get

$$
\begin{aligned}
F_{x^{l}}= & \frac{1}{4}\left(A^{1 / 2}+B\right)^{-1 / 2} A^{-1 / 2}\left(A_{x^{l}}+2 A^{1 / 2} B_{x^{l}}\right) \\
F_{x^{k} y^{l}} y^{k}= & -\frac{1}{16}\left(A^{1 / 2}+B\right)^{-3 / 2} A^{-1}\left(A_{y^{l}}+2 A^{1 / 2} B_{y^{l}}\right)\left(A_{0}+2 A^{1 / 2} B_{0}\right) \\
& +\frac{1}{8}\left(A^{1 / 2}+B\right)^{-1 / 2} A^{-3 / 2}\left(-A_{y^{l}} A_{0}+2 A A_{x^{m} y^{l}} y^{m}+4 A^{3 / 2} B_{x^{m} y^{l}} y^{m}\right) .
\end{aligned}
$$

Then using the fact that $A$ is not a perfect square of a quadratic form and the equation $F_{x^{k} y^{l}} y^{k}-F_{x^{l}}=0$, we obtain (11) and (12).

Proposition 4.2 Assume that $A$ is irreducible and $B \neq 0 . F=\left(A^{1 / 2}+B\right)^{1 / 2}$ is projectively flat if and only if there is a 1-form $\eta$ such that

$$
\begin{gathered}
4\left(A-B^{2}\right)\left\{B_{x^{m} y^{k}} y^{k}-B_{x^{k}}-2 \eta B_{y^{k}}\right\}=\left(B_{0}-4 \eta B\right)\left(A-B^{2}\right)_{y^{k}} . \\
2\left(A_{x^{k}}-4 \eta_{y^{k}} A-\eta A_{y^{k}}\right)=2 B\left\{B_{x^{m} y^{k}} y^{m}-B_{x^{k}}-2 \eta B_{y^{k}}\right\}-\left(B_{0}-4 \eta B\right) B_{y^{k}} .
\end{gathered}
$$

Proof: Assume that $F$ is projectively flat. Then $A$ and $B$ satisfy (11) and (12) respectively. It follows from (11) that

$$
M_{k} A=\left(2 A B B_{y^{k}}+2 B^{2} A_{y^{k}}-3 A A_{y^{k}}\right) A_{0},
$$

where

$$
M_{k}:=4\left(B^{2}-A\right)\left(A_{x^{m}} y^{k} y^{m}-A_{x^{k}}\right)-2\left(B A_{y^{k}}-2 A B_{y^{k}}\right) B_{0} .
$$

First we claim that $A_{0}$ is divisible by $A$. If this is not true, then from (15), one can see that $2 A B B_{y^{k}}+2 B^{2} A_{y^{k}}-3 A A_{y^{k}}$ is divisible by $A$ since $A$ is irreducible. That is, there is a 3 -form $\omega_{k}$ such that

$$
2 A B B_{y^{k}}+2 B^{2} A_{y^{k}}-3 A A_{y^{k}}=\omega_{k} A .
$$

Rewriting the above equation as follows

$$
2 B^{2} A_{y^{k}}=\left(\omega_{k}+3 A_{y^{k}}-2 B B_{y^{k}}\right) A .
$$

The right hand side is divisible by $B^{2}$. This is impossible since $A$ is irreducible and $\operatorname{deg}\left(\omega_{k}+3 A_{y^{k}}-2 B B_{y^{k}}\right)=3$. This proves our claim.

Therefore $A_{0}$ is divisible by $A$. There is a 1 -form $\eta$ such that

$$
A_{0}=8 \eta A \text {. }
$$

Rewrite (12) as follows

$$
B_{x^{m} y^{k}} y^{k}-B_{x^{k}}=\frac{A_{0}}{4 A} B_{y^{k}}-\frac{B A_{0}-2 A B_{0}}{2 A} Y_{k} .
$$


Plugging $A_{0}=8 \eta A$ into (17) yields (13).

Rewrite (11) as follows

$$
A_{x^{m} y^{k}} y^{m}-A_{x^{k}}=\frac{A_{0}}{2 A} A_{y^{k}}+B_{0} B_{y^{k}}+\left(A_{0}-2 B B_{0}\right) Y_{k} .
$$

Plugging $A_{0}=8 \eta A$ into (18) yields

$$
2\left(A-B^{2}\right)\left\{2\left(A_{x^{k}}-4 \eta_{y^{k}} A-\eta A_{y^{k}}\right)+\left(B_{0}-4 \eta B\right) B_{y^{k}}\right\}=B\left(B_{0}-4 \eta B\right)\left(A-B^{2}\right)_{y^{k}}
$$

Then (14) follows from (13) and (19).

It is easy to prove that the converse is true too.

Q.E.D.

Proof of Theorem 1.2: Assume that $F=\left(A^{1 / 2}+B\right)^{1 / 2}$ is projectively flat. Then by Proposition 4.2, (13) and (14) hold.

The right side of (13) is divisible by $A-B^{2}$. Since $A-B^{2}$ is irreducible and $\operatorname{deg}\left[\left(A-B^{2}\right)_{y^{k}}\right]=3$, we conclude

$$
B_{0}=4 \eta B
$$

Contracting (14) with $y^{k}$ yields

$$
A_{0}=8 \eta A \text {. }
$$

By a direct computation, we get the spray coefficients $G^{i}=P y^{i}$ with

$$
P=\frac{A_{0}+2 A^{1 / 2} B_{0}}{8 A^{1 / 2}\left(A^{1 / 2}+B\right)}=\frac{8 \eta A+8 \eta A^{1 / 2} B}{8 A^{1 / 2}\left(A^{1 / 2}+B\right)}=\eta .
$$

Thus $F$ is a Berwald metric.

In dimension $n \geq 3$, by Theorem 2.1, every Berwald metric of non-zero scalar flag curvature must be Riemannian. Then $B=0$ and $A$ is a perfect square of a Riemannian metric. This contradicts our assumption. Thus $\mathbf{K}=0$ and $F$ is locally Minkowskian.

Q.E.D.

\section{References}

[1] Z. Shen, Projectively flat Finsler metrics of constant flag curvature, Trans. Amer. Math. Soc. 355(4) (2003), 1713-1728.

[2] Z. Shen, On projectively flat (,)-metrics, Canadian Mathematical Bulletin, to appear.

[3] L. Berwald, Über die n-dimensionalen Geometrien konstanter Krümmung, in denen die Geraden die kürzesten sind, Math. Z. 30(1929), 449-469. 
[4] L. Berwald, On Finsler and Cartan geometries III, Two-dimensional Finsler spaces with vectilinear extremals, Ann. of Math. 42(1941), 84-112.

[5] S. Numata, On Landsberg spaces of scalar curvature, J. Korea Math. Soc. 12(1975), 97-100.

[6] D.G. Pavlov (ed.), Space-Time Structure, Collected papers, TETRU, 2006.

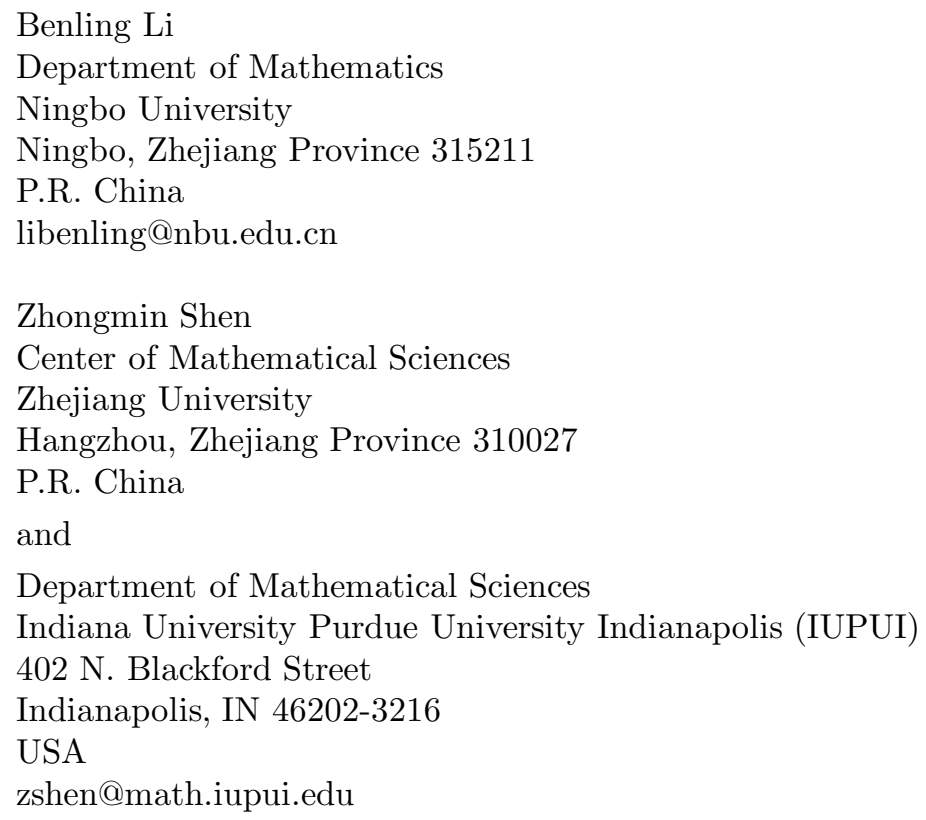

